\title{
Caracterización Molecular de Aislados Centroamericanos de Beauveria bassiana para el Control de la Broca del Café
}

\author{
Gustavo Fontecha *, Rogelio Trabanino †, Beatriz Pérez-Borrero ‡, \\ Pablo Catalán ₹, Estela Aguilar ¥, Francisco Javier Gallego ‡, \\ Ana Margarita Figueiras ‡, Césa Benito ‡
}

\section{RESUMEN}

La plaga más importante para los cultivos de café en Honduras es la "Broca" (Hypothenemus hampei). Beauveria bassiana es un hongo utilizado como controlador biológico de este insecto. Los aislamientos locales de este hongo han sido evaluados hasta ahora de manera tradicional, pero no han sido caracterizados genéticamente para establecer variabilidad y relaciones filogenéticas entre aislados y relacionar esto con su patogenicidad. Nuestro estudio explora la aplicación potencial de la diversidad genética de once aislados centroamericanos de $B$. bassiana mediante tres marcadores moleculares: Intermicrosatélites, secuencias de SSR e ITS. La infectividad de estos aislados fue evaluada en laboratorio y en el campo. Los aislados analizados revelaron baja diversidad genética con los marcadores usados. No hubo diferencias estadísticamente significativas en la capacidad infectiva de la broca.

A pesar de ello, BB-XN demostró ser la más eficaz como patógeno tanto en el laboratorio como en las plantaciones de café. Aunque no fue posible distinguir los aislamientos mediante análisis ITS, encontramos que tres cebadores ISSR fueron capaces de distinguir individualmente el perfil genético de todos ellos. Mediante la secuenciación de SSR obtuvimos un dendrograma con una estructura idéntica a la obtenida mediante ISSR; por tanto ambos marcadores son igualmente capaces de establecer las relaciones filogenéticas entre los aislados del hongo. En conclusión, hemos optimizado un conjunto de marcadores capaces de determinar la variabilidad entre aislamientos regionales de $B$. bassiana. Lo anterior constituye el primer paso para su aplicación en la distinción de genotipos del hongo y su ulterior comercialización.

\footnotetext{
* Universidad Nacional Autónoma de Honduras. Escuela de Microbiología. Tegucigalpa, Honduras. Autor de referencia. gustavofontechen@unah.edu.hn.

† Escuela Agrícola Panamericana. Laboratorio de Control Biológico. Tegucigalpa, Honduras

‡ Universidad Complutense de Madrid. Departamento de Genética. España.
} 
Palabras clave: Beauveria bassiana, Hypothenemus hampei, Honduras, Centro América, ISSR, ITS.

\section{ABSTRACT}

The most important pest for coffee crops in Honduras is the berry borer Hypothenemus hampei. Beauveria bassiana is a fungus used in biological control of this insect. Local fungi isolates have been evaluated in a traditional way, but they haven't been characterized genetically in order to establish the relationship between isolates and its pathogenicity. Our study explored the potential application of genetic diversity of Central American $B$. bassiana isolates using Intermicrosatellite markers, SSR sequences and ITS. These isolates were also analyzed by their infectivity under laboratory and field conditions. All the isolates obtained from the Central American region show low genetic diversity and there were not significant differences in their infective capabilities over the coffee berry borer.

Besides that, BB-XN had a better performance as a pathogen both in laboratory as in field conditions. Although it was not possible to distinguish the isolates by the ITS approach, we found three ISSR primers which alone could distinguish all the Central American isolates. Through these markers is possible to distinguish the identity of each isolate. The sequence analysis of SSR produced a dendrogram with exactly the same structure than the tree obtained from the amplifications with ISSR. Therefore SSR sequences are as reliable as the analysis of ISSR in order to establish phylogenetic relationships between isolates. In conclusion, we have optimized a set of markers in order to determine variability of regional Beauveria's isolates and this is the first step to apply them for distinguishing between genotypes and its commercialization.

Key words: Beauveria bassiana, Hypothenemus hampei, Honduras, Central America, ISSR, ITS. 


\section{INTRODUCCIÓN}

El hongo Beauveria bassiana es una especie endofítica, ubicua y entomopatógena, con un amplio rango de hospederos. Se le define como un hongo benéfico por su capacidad de infectar especies de Lepidoptera, Coleoptera e Hymenoptera, definidas como plagas agrícolas. Su potencial en el control biológico ha sido utilizado aplicando aislados locales del hongo; colectados del suelo o de insectos hospederos muertos a partir de diferentes áreas geográficas. Este hongo ha sido empleado en muchas plantaciones de café en Latinoamérica para controlar las poblaciones larvales de Hypothenemus hampei (Ferr.), conocido como "broca del café".

Actualmente se reconoce que $B$. bassiana es un complejo biológico compuesto por varias especies filogenéticamente asociadas a Ascomycotina. Tradicionalmente, la caracterización de $B$. bassiana está basada en la descripción y en la medida de estructuras de interés agronómico, en su fisiología , patogénicas , y en estudios isoenzimáticos. Más recientemente la caracterización de $B$. bassiana ha sido enriquecida mediante marcadores de ADN: RFLP, AFLP, RAPD, ITS, y Microsatélites (SSRs). La variación molecular encontrada entre aislados de Beauveria ha sido relacionada con el rango de insectos hospederos y se ha evaluado mediante varias técnicas moleculares. Los ISSR (inter-simple sequence repeats) son productos de PCR obtenidos usando cebadores basados en repeticiones de nucleótidos. Estos ISSR se basan en la amplificación de regiones entre microsatélites orientados en sentido inverso y aledaños entre sí. Los ISSR se han empleado con diferentes propósitos como la identificación de cultivares de plantas, y la detección de la variabilidad genética de gran variedad de organismos . Recientemente, Ios ISSR han sido usados para estudiar algunos aislados de Beauveria bassiana.

Dado que los aislados circulantes de Beauveria, tanto silvestres como comerciales, poseen distintas capacidades infectivas y son morfológicamente indistinguibles; se consideró conveniente caracterizarlos molecularmente utilizando tres tipos de marcadores genéticos: Intermicrosatélites (PCR-ISSR), secuencias de SSR e ITS. También se correlacionaron sus perfiles genéticos con su capacidad infectiva bajo condiciones de laboratorio y de campo. En este estudio hemos logrado distinguir entre genotipos de Beauveria de diferente origen geográfico, incluyendo once aislados centroamericanos. Al mismo tiempo hemos establecido las relaciones filogenéticas entre los aislados estudiados y la manera en la que estos datos se correlacionan con su efectividad como herramientas en el control biológico de la broca del café. La importancia última de este trabajo radica en que la selección de 
cepas virulentas y genéticamente bien caracterizadas de $B$. bassiana es la piedra angular en las investigaciones para la producción comercial de este hongo entomopatógeno como controlador biológico.

\section{MATERIALES Y MÉTODOS}

\section{Aislados de Beauveria bassiana}

Se seleccionaron once aislados de Beauveria del Banco-Cepario de la Escuela Agrícola Panamericana-Zamorano (EAP) en Honduras. Algunos de los aislamientos estudiados corresponden a aquellos que actualmente se están utilizando en las plantaciones de café cercanas a la Universidad o son aislamientos obtenidos en Bancos de países vecinos (Cuadro 1). Cinco de los aislados seleccionados se utilizan comercialmente en Guatemala, Honduras y Costa Rica; mientras que los seis restantes provienen de Bancos que aún no se explotan comercialmente.

Además se incluyeron en los análisis de ISSR once aislados externos procedentes de Cuba, Guadalupe, India, Bulgaria y Estados Unidos. Lo anterior con el fin de comparar su variabilidad genética respecto a los hongos centroamericanos. Estos últimos no se describen en detalle en este estudio ya que no se sometieron a estudios fenotípicos.

Cuadro 1. Aislamientos de Beauveria bassiana analizados

\begin{tabular}{|c|c|c|}
\hline Nombre & País de Procedencia & Orígen \\
\hline BB-Nic & Nicaragua & Cepario \\
\hline BB-084 & Costa Rica. CATIE & Cepario \\
\hline BB-089 & Costa Rica. CATIE & Cepario \\
\hline Disagro & Guatemala & Comercial \\
\hline Teraboveria & Guatemala & Comercial \\
\hline BB-0415 & Costa Rica. CATIE & Cepario \\
\hline BB-XN & Costa Rica. CATIE & Cepario \\
\hline BB-Zam & honduras. EAP & Comercial \\
\hline Cengicaña & Guatemala & Comercial \\
\hline BB-Sal & El Salvador & Cepario \\
\hline BB-CR1 & Costa Rica & Comercial \\
\hline
\end{tabular}




\section{Análisis fenotípico}

Los análisis de virulencia del hongo se realizaron de acuerdo con el método descrito por Gonzales et al 1993 en la Escuela Agrícola Panamericana (EAP). Estos insectos adultos del coleóptero se obtuvieron de una colonia mantenida bajo condiciones de laboratorio . Los insectos fueron inoculados por inmersión con suspensiones de $10 \mathrm{ml}\left(1 \times 10^{6}\right.$ conidios $\left.\mathrm{ml}^{-1}\right)$ y transferidos a contenedores individuales bajo temperatura y condiciones de humedad constantes.

La mortalidad debida a los hongos fue registrada a intervalos de 24 horas durante 8 días. Los insectos afectados fueron cultivados en medio SDA y el crecimiento del micelio fue subcultivado en el mismo medio. Los conidios de Beauveria se almacenaron en glicerol $\mathrm{a}-80^{\circ} \mathrm{C}$.

La primera fase de la investigación se realizó en condiciones de laboratorio donde se evaluó la mortalidad de los adultos de $H$. hampei después de ser sumergidos en una solución de esporas de $B$. bassiana. La investigación se complementó con una segunda fase de campo en una plantación de café. Dicha plantación se ubica en la Hacienda Santa Elisa, a $5 \mathrm{~km}$ de la ciudad de Danlí, en el sureste de Honduras, a una altura de $800 \mathrm{msnm}$ y una temperatura promedio anual de $25^{\circ} \mathrm{C}$. La variedad del café sembrado fue Caturra a una distancia de $2 \mathrm{~m}$ entre planta por $1.5 \mathrm{~m}$ entre surco. $\mathrm{La}$ evaluación en campo consistió en aplicar directamente esporas de $B$. bassiana en la plantación de café para evaluar su control.

\section{Muestreo de insectos de la broca y tratamientos}

En total se realizaron 12 tratamientos para once aislados de $B$. bassiana más un tratamiento testigo con agua. Cada tratamiento se efectuó 4 veces, para un total de 52 unidades experimentales. Cada unidad experimental consistió en una placa Petri con 20 brocas de café adultos.

\section{Prueba de concentración y viabilidad de los aislados}

Se contó la cantidad de unidades formadoras de colonia (UFC/g) y la viabilidad de cada aislado de acuerdo a la metodología descrita por Sandoval 1997 . La viabilidad de cada tratamiento se determinó con el conteo de un mínimo de 100 esporas, tomando como espora germinada aquella que presentó desarrollo del tubo germinativo. 


\section{Aplicación de los tratamientos}

Se utilizó el método de inmersión de la broca, en el cual se colocaron 20 brocas en una malla de tela y se sumergieron en una solución de $500 \mathrm{ml}$ de cloro $(0,05 \%)$ durante dos minutos. Seguidamente se sumergieron en una suspensión de conidios de $B$. bassiana, a razón de $3.33 \times 10^{5}$ esporas $\mathrm{ml}^{-1}$, durante un minuto. Ya inoculadas las 20 brocas con $B$. bassiana se colocaron en una placa Petri con papel filtro humedecido. Cada placa Petri se colocó en un cuarto a una temperatura promedio de $28^{\circ} \mathrm{C}$.

El ensayo se evaluó a los 7 días de ser inoculados los insectos. Para determinar el porcentaje de mortalidad se contó el número de adultos muertos y vivos de broca de café. A los 15 días se repitió la evaluación, esta vez contando el número de brocas vivas, muertas y con micelio (esporuladas) del hongo entomopatógeno.

\section{Análisis estadístico}

Para el análisis estadístico de los resultados se usó el paquete estadístico MINTAB. Se realizó un Análisis de varianza (ANDEVA) usando un Modelo Lineal General (GLM) y una separación de medias Duncan con un nivel de significancia de 0,05.

\section{Muestreo de campo y tratamientos}

Antes de las aplicaciones se realizó un muestreo para determinar el porcentaje de infestación en las plantaciones. Si la infestación era mayor o igual al 5\% era necesario aplicar el tratamiento. Se evaluó el efecto de once aislados de $B$. bassiana en campo a una concentración de $1.44 \times 10^{10}$ esporas por hectárea (4.8 $\times$ $10^{7}$ esporas/planta). Se incluyeron dos testigos, uno con agua potable y otro con Thiodan® (Endosulfan) a una dosificación de 4 litros por hectárea. La solución de esporas para cada tratamiento se aplicó a los cafetos dirigidos a fruto, sobre una parcela de $10 \mathrm{~m}^{2}$, con aproximadamente 30 cafetos ( 5 surcos $\times 6$ plantas). La aplicación se realizó durante la hora más fresca de la tarde. Se estableció un tiempo de aplicación de 1 min y 43 seg por planta.

Quince días después de la aplicación se recolectaron los frutos brocados y se trasladaron al laboratorio para evaluar el número total de brocas muertas y parasitadas por el hongo. Las brocas extraídas de los granos se colocaron en placas Petri con papel humedecido en un cuarto de incubación a $28^{\circ} \mathrm{C}$ durante cinco días. 


\section{Marcadores de ISSR - PCR}

Se analizaron en total once aislados de Beauveria bassiana (cuadro 1) mediante ISSR-PCR. Los aislamientos del hongo fueron cultivados en placas de Petri a $27^{\circ} \mathrm{C}$ por varios días en medio PDA hasta la producción suficiente del micelio.

\section{Extracción del ADN}

El micelio obtenido se utilizó para la extracción del ADN de acuerdo al método de aislamiento de ADN a pequeña escala, Wizard® Genomic ADN Purification Kit, Promega.

\section{Cebadores utilizados en las PCR}

Se analizaron dieciséis cebadores ISSR en este trabajo. Estos oligonucleótidos se obtuvieron del ISSR Primer Set 100/9 (University of British Columbia) y fueron seleccionados de acuerdo al número y consistencia de los fragmentos amplificados en un trabajo previo (Cuadro 2).

Cuadro 2. ISSR primer set $\# 9$ UBC y su respectiva secuencia de oligonucleótidos. Clave: $\boldsymbol{B}=\mathrm{C} / \mathrm{G} / \mathrm{T} ; \boldsymbol{H}=\mathrm{A} / \mathrm{C} / \mathrm{T} ; \boldsymbol{R}=\mathrm{A} / \mathrm{G} ; \mathrm{Y}=\mathrm{T} / \mathrm{C} ; \mathrm{D}=\mathrm{G} / \mathrm{T} / \mathrm{A}$

\begin{tabular}{|c|c|c|c|}
\hline Cebador & Secuencia & Cebador & Secuencia \\
\hline 808 & (AG) 8C & 873 & (GACA) 4 \\
\hline 809 & (AG) 8G & 885 & BHB(GA) 7 \\
810 & (GA) 8T & 888 & BDB(CA) 7 \\
828 & (TG) 8A & 889 & DBD(AC) 7 \\
842 & (GA) 8YG & GG 808 & GC(AG) 8C \\
846 & (CA) 8RT & GG 821 & GG (GT) 8T \\
849 & (GT) 8YA & GG 846 & GG (CA) 8RT \\
\hline 850 & (GT) 8YC & GG 873 & GG(GACA) 4 \\
\hline
\end{tabular}

\section{Amplificación de secuencias, poder de resolución y perfil de reproducibilidad de bandas}

Para la reacción de amplificación adaptamos el protocolo de Estrada et al 2007 . Utilizamos el ADN extraído del micelio y cada reacción de amplificación (volumen final de $21 \mu \mathrm{l}$ ) contenía $2 \mu \mathrm{I}$ del extracto del ADN genómico (10 ng/ì I), $1 \mu \mathrm{I}$ del 
cebador correspondiente (5 ì M), $10 \mu \mathrm{I}$ de PCR Master Mix 2X (Promega) y $8 \mu \mathrm{I}$ de agua ultra pura, libre de nucleasas (Promega). El programa de amplificación consistió de un paso inicial de 5 min a $94^{\circ} \mathrm{C}$, seguido por 45 ciclos de $30 \mathrm{sa} 94^{\circ} \mathrm{C}, 45$ s a $52^{\circ} \mathrm{C}, 2$ min a $72^{\circ} \mathrm{C}$, y por último una extensión de 6 min a $72^{\circ} \mathrm{C}$. Las reacciones de amplificación fueron almacenadas a $4^{\circ} \mathrm{C}$ hasta su separación por electroforesis. Los productos de PCR fueron analizados en geles de agarosa- bromuro de etidio al $1.5 \%$ y corridos a $90 \mathrm{~V}$ por 2 horas.

Las amplificaciones de ADN de todos los aislados de Beauveria bassiana fueron analizadas por triplicado con cada uno de los cebadores ISSR para determinar la reproducibilidad de las bandas. Además se amplificaron y secuenciaron las muestras con ocho cebadores específicos de genoma de Beauveria (Cuadro 3). Estos cebadores fueron diseñados en el Laboratorio de César Benito en la Universidad Complutense de Madrid, a partir de secuencias previamente amplificadas de ISSR. Las condiciones de amplificación son semejantes a las descritas para el análisis de microsatélites.

Cuadro 3. Secuencias de cebadores utilizados para amplificar regiones SSR de Beauveria bassiana.

\begin{tabular}{|c|l|l|}
\hline Nombre & \multicolumn{1}{|c|}{ Secuencia Cebador $\mathbf{1}$} & \multicolumn{1}{c|}{ Secuencia Cebador $\mathbf{2}$} \\
\hline Beau-1 & GCGCAGGGATGCCAAGGTCTC & GCCGGTAAAACGAGGGTAGA \\
\hline Beau-2a & AGTTCGTGTCTCATCGGCTAC & AGCTTATCGCGTACCAGTT \\
\hline Beau-2b & GAGAGGCAGGAAAGGCGATG & GGCGAGCGAACGTCTTTG \\
\hline Beau-2c & GCGCAATGTTTCGTAGAAG & TACATACGCAAGTCCGATGAG \\
\hline Beau-3 & GGAGGATCTCAAATCCCTAAT & CGTCGTCGTCGTCAAATG \\
\hline Beau-32 & GGAGTTCGTACGCACC & AAGTCCACGTCCTTACTA \\
\hline Beau-52 & GCGGTGGAAAGATATCAA & AGATGCCAGAGTGTTCGATCA \\
\hline Beau-72 & CGGCCGGACTGGTTACTAGAT & TTCCACCGCGCATCAAT \\
\hline
\end{tabular}

\section{Análisis de las amplificaciones}

A las matrices binarias creadas para el análisis de los aislados de Beauveria bassiana se les proporcionó el valor de (1) para presencia de bandas y (0) para ausencia (bandas con el mismo tamaño). Las relaciones filogenéticas entre aislados fueron establecidas utilizando los programas NTSYSpc y WinBoot. Para ello se usaron distintos índices (SM, DICE y Jacard) y el método de agrupamiento UPGMA con un Bootstrap de 10,000. 


\section{Amplificación de dos regiones ITS ribosomales}

Se amplificaron dos regiones ITS del hongo utilizando dos juegos de cebadores: ITS1F-ITS2 e ITS3-ITS4 (Fig. 1).

Figura 1. Esquema parcial del gen ADN ribosomal y sus espaciadores internos ITS1 e ITS2. Las flechas indican la posición de hibridación de los cebadores.

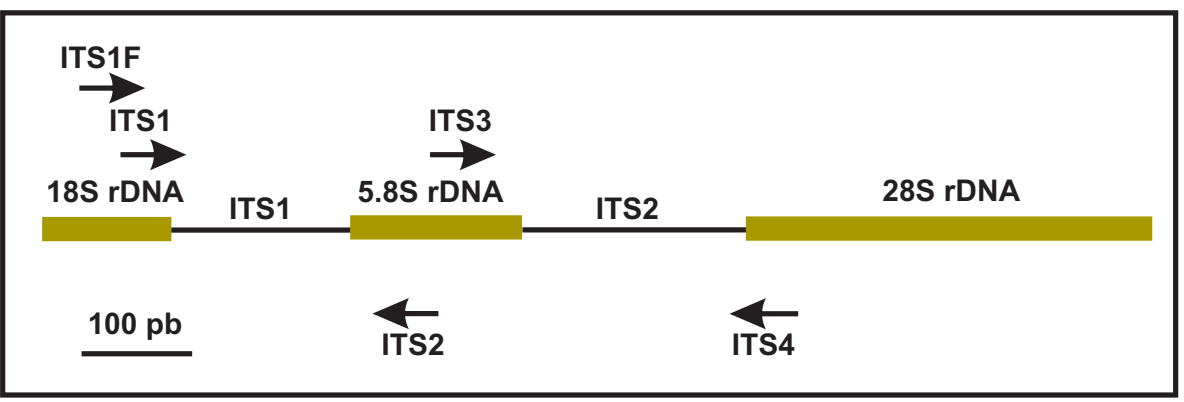

Las amplificaciones se llevaron a cabo en un volumen final de $20 \mu \mathrm{l}$ en un Termociclador Veriti ${ }^{\mathrm{TM}}$ (Applied Biosystems). La mezcla de reacción contenía 10 ul de PCR Master Mix 2X (Promega), $1 \mu \mathrm{I}$ de cada cebador $10 \mu \mathrm{M}$, agua libre de nucleasas y $1 \mu \mathrm{I}$ de ADN molde $10 \mathrm{ng} / \mu \mathrm{l}$. El programa de la amplificación fue el siguiente: $94^{\circ} \mathrm{C}$ por $1 \mathrm{~min}, 35$ ciclos a $94^{\circ} \mathrm{C}$ por $1 \mathrm{~min}, 55^{\circ} \mathrm{C}$ por $2 \mathrm{~min}, 72^{\circ} \mathrm{C}$ por 2 min, yuna extensión final $\mathrm{a} 72^{\circ} \mathrm{C}$ por $10 \mathrm{~min}$.

Los productos se visualizaron después de una electroforesis de agarosa $1 \%$ con bromuro de etidio. Los productos de amplificación se sometieron a PCR-RFLP usando Haelll, Hinfl, Ndel, Hhaly Alul.

La mezcla de reacción de la digestión para un volumen de $20 \mu$ I contenía: 15,3 $\mu$ I de agua libre de nucleasas, $2 \mu \mathrm{I}$ de buffer $10 \mathrm{X}, 0.2 \mu \mathrm{I}$ de BSA $10 \mu \mathrm{g}, 2.0 \mu \mathrm{I}$ del producto de PCR y después de mezclarlo se agregó $0.5 \mu \mathrm{I}$ de la enzima $10 \mathrm{U} / \mu \mathrm{l}$. Se incubó a $37^{\circ} \mathrm{C}$ durante 4 horas y se visualizó el resultado en un gel de agarosa al $2 \%$.

\section{Secuenciación de amplicones}

Los amplicones de las regiones ITS fueron enviados a Macrogen (EE.UU) para su secuenciación. Las secuencias de las regiones SSR fueron obtenidas en las Instalaciones de la Facultad de Biología de la Universidad Complutense. 


\section{RESULTADOSYDISCUSIÓN}

\section{Resultados de mortalidad de broca de café y porcentaje de parasitismo}

La mayor mortalidad en el laboratorio se observó en el tratamiento con BB-XN. El aislado que presentó el menor porcentaje de mortalidad fue Cengicaña. Después de incubar durante 8 días más, la evaluación de parasitismo indicó que las BB-XN y BB- Sal tuvieron el mayor porcentaje de brocas parasitadas pero fueron estadísticamente iguales al resto de aislados.

BB-Nic, BB-CR1 y Disagro tuvieron los porcentajes más bajos de parasitismo. Esto indica que no existe diferencia estadísticamente significativa entre los tratamientos en el porcentaje de mortalidad a los 7 días $(P=0.063$ ) (Cuadro 4).

Cuadro 4. Evaluación del porcentaje de mortalidad y porcentaje de parasitismo de broca de café con Beauveria bassiana.

\begin{tabular}{|c|c|c|c|}
\hline Tratamiento & $\begin{array}{c}\text { Aislados de } \\
\text { B. bassiana }\end{array}$ & $\begin{array}{c}\text { Mortalidad a } \\
\text { los 7 dias (\%) }\end{array}$ & $\begin{array}{c}\text { Brocas esporuladas } \\
\text { a los 15 dias (\%) }\end{array}$ \\
\hline 1 & BB-089 & 59.078 & 64.8 \\
\hline 2 & BN-XN & 98.438 & 93.9 \\
\hline 3 & BB-Nic & 76.563 & 65.2 \\
\hline 4 & BB-Sal & 81.250 & 94.6 \\
\hline 5 & BB-0415 & 75.219 & 83.3 \\
\hline 6 & Teraboveria & 88.440 & 74.1 \\
\hline 7 & BB-Zam & 82.353 & 63.6 \\
\hline 8 & BB-089 & 79.239 & 69.9 \\
\hline 9 & BB-CR1 & 57.750 & 75.4 \\
\hline 10 & Cengicaña & 50.975 & 61.1 \\
\hline 11 & Disagro & 52.675 & 57.9 \\
\hline 12 & agua & 56.380 & $4.4^{*}$ \\
\hline Probabilidad & & $\mathbf{0 . 0 6 3}$ & $\mathbf{0 . 0 0 0}$ \\
\hline
\end{tabular}

* Significativamente diferentes $(P \leq 0.05)$ 
Cuadro 5. Porcentaje de brocas parasitadas con B. bassiana recolectadas en granos de café previamente tratados.

\begin{tabular}{|c|c|}
\hline Aislado de Beauveria bassiana & Porcentaje brocas parasitarias \\
\hline BB-Nic & 18.40 \\
\hline BB-Sal & 23.10 \\
\hline BB-0089 & 31.15 \\
\hline BB-XN & 39.40 \\
\hline BB-084 & 21.65 \\
\hline Teraboveria & 37.82 \\
\hline BB-Zam & 20.05 \\
\hline BB-0415 & 30.27 \\
\hline Cengicaña & 36.20 \\
\hline BB-CR1 & 34.70 \\
\hline Disagro & 20.05 \\
Agua & 0.00 \\
Químico & 0.00 \\
\hline Probabilidad & $\mathbf{P}=\mathbf{0 . 1 4 4}$ \\
\hline
\end{tabular}

\section{Eficacia de Beauveria en los análisis de campo}

Los resultados obtenidos en el laboratorio indican que los aislados de $B$. bassiana tienen una viabilidad y una patogenicidad semejantes a lo que refieren algunos artículos de la literatura internacional; sin embargo, ésto no necesariamente asegura que en condiciones de campo estos aislados sean igualmente eficaces ya que los factores ambientales y factores mecánicos de la aplicación podrían afectar directamente el resultado final del desarrollo y permanencia del hongo. Esta idea es compatible con el número de brocas parasitadas a los 7 días después de aplicar el hongo en el campo a los granos semi-maduros.

Estos experimentos reflejan una reducción considerable en la eficacia de los resultados de laboratorio respecto a los del campo. En estos ensayos de campo se observó una mayor mortalidad en los tratamientos con el aislado de B. bassiana BB$\mathrm{XN}$, mientras que el aislamiento que produjo menor mortalidad fue el originario de Nicaragua. El análisis estadístico (con una probabilidad de $\mathrm{P}=0.144$ ) indica que tampoco existe diferencia entre los tratamientos, por tanto, no es posible seleccionar con certeza algún aislado de acuerdo solamente a su eficacia en el campo. El tratamiento con agua y el tratamiento con químico no presentaron esporulación de ningún tipo (Cuadro 5). 
El aislado BB-XN de Costa Rica mostró el porcentaje más parecido al del estudio realizado por Bustillo en el año 1999, quien con una sola aplicación informó un control de broca en condiciones de campo de 36\%. Sin embargo, el amplio rango de eficacia para parasitar brocas por los aislados analizados (18.4\% - 39.4\%) es un dato congruente con los hallazgos de otros investigadores, incluso utilizando otros insectos plaga como diana.

Estas variaciones en la capacidad infectiva bien pueden reflejar el efecto que el ambiente ejerce sobre el comportamiento de este hongo, sumado a los fenotipos individuales de cada aislado. En ese mismo estudio se demostró que los niveles de infección aumentaron hasta un 69\% cuando se asperjó el hongo seis veces. Estudios similares de control de otras especies de insectos, usando Beauveria, revelan que la eficacia oscila grandemente desde 10\% hasta $65 \%$; dependiendo de los aislados del hongo empleados, de la técnica de aplicación de los conidios sobre las plagas o de la época del año en la que los artrópodos son más susceptibles .

Sería razonable pensar pues que asperjar repetidamente suspensiones mixtas del hongo pudiera compensar, de alguna manera, las variaciones en la eficacia de parasitismo que cada uno de los aislados manifiesta, ya que el control de las condiciones ambientales está fuera del alcance del usuario. A pesar de todas estas consideraciones, parece claro que BB-XN tuvo el mejor rendimiento en condiciones de laboratorio al igual que en condiciones de campo (aunque la significancia estadística no sea contundente), lo que sugiere que genéticamente podría ser la más virulenta contra la broca de café.

\section{PCR-RFLP y secuencias de ITS en los aislados procedentes de Centroamérica}

Como primer marcador molecular, se amplificaron dos regiones espaciadoras internas (ITS) de genes ribosomales de los once aislados procedentes de América Central. Lo anterior con el objeto de detectar variabilidad mediante diferencias en los tamaños de los productos de amplificación. Sin embargo, al igual que en otras publicaciones los once aislados analizados presentaron tamaños idénticos (Figura 2).

Figura 2. PCR ITS1F-ITS2 (A) e ITS3-ITS4 (B).
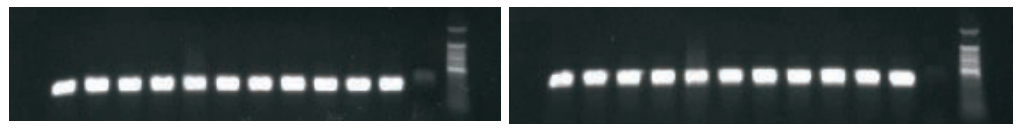
El segundo enfoque utilizado para poner de manifiesto la variabilidad en estas secuencias fue la digestión de los amplicones con cinco enzimas de restricción (PCR-RFLP) de ambas regiones ITS. El nivel de variación detectado fue nulo (Figura 3). Ninguna de las cinco enzimas de restricción fue capaz de detectar diferencias entre los amplicones, y en consecuencia, no fue posible distinguir entre los aislados analizados con esta técnica. En el estudio de Gaitán y colaboradores se analizaron, mediante ITS, más de 90 aislados procedentes de diferentes regiones del mundo y sólo fueron capaces de evidenciar polimorfismos al aplicar dos enzimas (Alul y $M s p /)$. Otras seis enzimas produjeron patrones idénticos de corte o no encontraron diana en los amplicones. Trabajos recientes demuestran que las secuencias de ITS no parecen ser buenas opciones para evidenciar diferencias filogeográficas en este hongo. Ghikas y colaboradores opinan que es más conveniente utilizar regiones espaciadoras mitocondriales (nad3-atp9 y atp6-rns) para este efecto. Becerra y colaboradores realizaron un estudio con cepas chilenas del hongo y añaden que aunque el PCR-RFLP de las regiones ITS 1 y 2 detectó diversidad genética en esta especie, los aislamientos no fueron completamente discriminados entre ellos, al comparar esa técnica con análisis que implican todo el genoma, como los RAPD. Tampoco para estos autores los ITS establecieron la relación entre los aislamientos y su origen geográfico, así como tampoco pudieron discriminar entre aislamientos.

Al no poder establecer diversidad entre los aislados centroamericanos de Beauveria decidimos secuenciar estos amplicones. En el ITS1 se encontraron solamente dos SNP en un total de $281 \mathrm{pb}$. En el ITS2 se detectaron cuatro SNP y un INDEL de un solo nucleótido en una secuencia total de 381pb. El dendrograma obtenido con estas dos secuencias de ITS (Figura 4) demuestra que tampoco la secuenciación de las regiones espaciadoras ribosomales permitió diferenciar los once aislados estudiados.

Figura 3. Dos ensayos de restricción de las regiones ITS evidenciando la falta de variabilidad entre los once aislados.
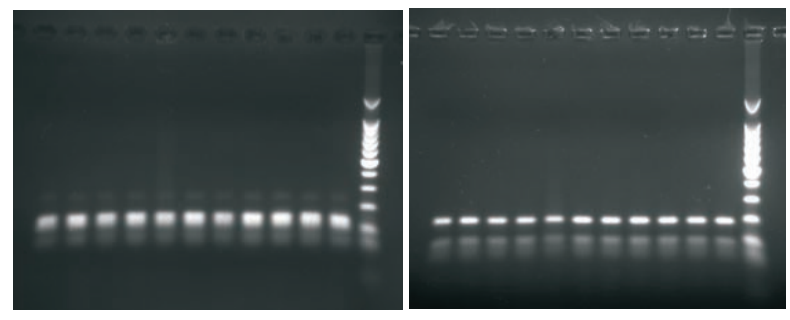

Sin embargo, dado que la identidad con las secuencias previamente publicadas para Beauveria bassiana en las bases de datos (NCBI) fue del $100 \%$, se puede 
concluir que al menos estos dos ITS corroboran que todas los aislados estudiados pertenecen a la especie Beauveria bassiana.

Figura 4. Dendrograma obtenido a partir de las dos secuencias de las regiones ITS ribosomales

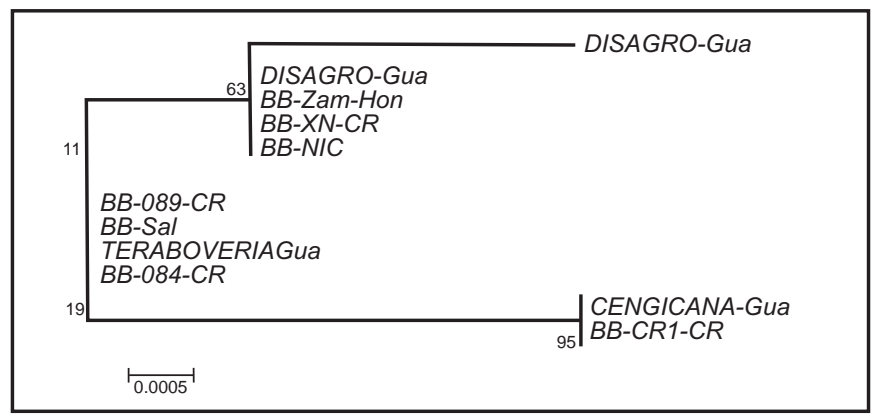

\section{Intermicrosatélites (ISSR)}

Se analizaron 22 aislados de Beauveria bassiana de distinta procedencia, once de Centro América y once procedentes de Cuba, Guadalupe, India, Bulgaria y USA. Para ello se amplificaron diferentes Intermicrosatélites (ISSRs) en ADN genómico y, por otro lado, se obtuvo la secuencia de ocho ISSRs diferentes. En los once aislados Centroamericanos se obtuvieron 230 productos de amplificación distintos usando 16 cebadores de ISSRs. Con los 16 cebadores empleados fue posible identificar los once aislados (Figura 5). El número total de bandas (NTB) amplificadas con cada cebador osciló entre 25 y 8, el número de bandas polimórficas (NBP) entre 6 y 25, el porcentaje de bandas polimórficas (P\%) varió entre el $64,28 \%$ y el $100 \%$. El número observado de genotipos (NG) osciló entre 4 y 11 y el número de bandas exclusivas (NBE, que solamente se observan en un aislado o que solamente están ausentes en un aislado), osciló entre 0 y 6 . Se encontraron tres cebadores que por sí solos podían distinguir los once aislados: 810,842 y 846.

Figura 5. Amplificación de ISSR utilizando el cebador 846.

Cebador 846

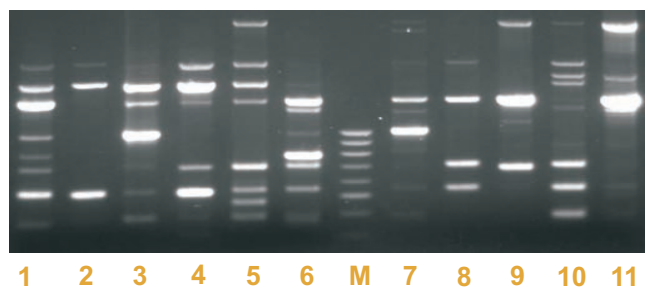


En el cuadro 6 se resumen los resultados obtenidos con cada cebador y se indican los resultados conjuntos. Se puede observar que el porcentaje de bandas polimórficas es bastante alto, 91,73\%. Los resultados filogenéticos obtenidos, al analizar los hongos procedentes de América Central mediante los 230 productos de ISSR, indican que no existe una relación entre el origen geográfico de los hongos y las filogenias obtenidas. Dos de los aislados estudiados se separaron claramente del resto: Cengicaña de Guatemala y BB-CR1-CR de Costa Rica. Este mismo patrón se evidenció con el análisis de las secuencias ITS, lo que confirma el hecho de que ambos aislados están más alejados filogenéticamente del resto. Lamentablemente este resultado no está relacionado con el comportamiento patógeno de estos hongos.

Cuadro 6. Resultados de los perfiles de bandas de ISSR en los aislados de $B$. bassiana de origen centroamericano.

\begin{tabular}{|c|c|c|c|c|c|}
\hline Cebador & NTB & NBP & P\% & NG & NBE \\
\hline 808 & 15 & 15 & 100 & 8 & 1 \\
\hline 809 & 15 & 13 & 86.66 & 9 & 0 \\
\hline 810 & 16 & 16 & 100 & 11 & 6 \\
\hline 828 & 12 & 12 & 100 & 10 & 2 \\
\hline 842 & 25 & 25 & 100 & 11 & 6 \\
\hline 846 & 14 & 14 & 100 & 11 & 6 \\
\hline 849 & 10 & 10 & 100 & 6 & 4 \\
850 & 14 & 11 & 78.57 & 9 & 1 \\
\hline 873 & 14 & 9 & 64.28 & 4 & 1 \\
\hline 885 & 16 & 14 & 87.5 & 8 & 0 \\
888 & 14 & 13 & 92.85 & 4 & 0 \\
\hline 889 & 16 & 13 & 81.25 & 6 & 0 \\
\hline GG 808 & 18 & 18 & 100 & 10 & 1 \\
\hline GG 821 & 8 & 6 & 75.0 & 6 & 0 \\
\hline GG 846 & 9 & 8 & 88.88 & 6 & 1 \\
\hline GG 873 & 14 & 14 & 100 & 5 & 0 \\
\hline TOTAL & 230 & 211 & 91.73 & 124 & 29 \\
\hline
\end{tabular}

Y aunque no hay diferencias claras entre el resto de aislados, es posible mediante estos marcadores distinguir la identidad de cada uno de los aislamientos. A pesar de que estos marcadores no fueron capaces de discriminar el origen geográfico, estudios realizados con 110 aislamientos procedentes de diversos puntos del Medio Este y Asia Central, reflejan que los ISSR utilizados dieron indicios de 
agrupamientos correlacionados con el origen geográfico y mostraron una relativa diversidad genética entre algunos aislados[38]. Por otro lado; los aislamientos analizados mediante ISSR en la región central de China, y de procedencia geográficamente cercana, mostraron un nivel relativamente aceptable de diversidad intra-específica pero un bajo nivel de diferenciación entre poblaciones[25].

Nuestros resultados sugieren que estos marcadores son útiles para diferenciar aislamientos geográficamente distantes, pero no son ideales para poblaciones cercanas, aunque indiscutiblemente siguen siendo considerados como marcadores robustos para estudios epidemiológicos y ecológicos[26] .

Figura 6. Relaciones filogenéticas entre los aislamientos Centroamericanas de B. bassiana utilizando marcadores ISSR. Agrupación por UPGMA.

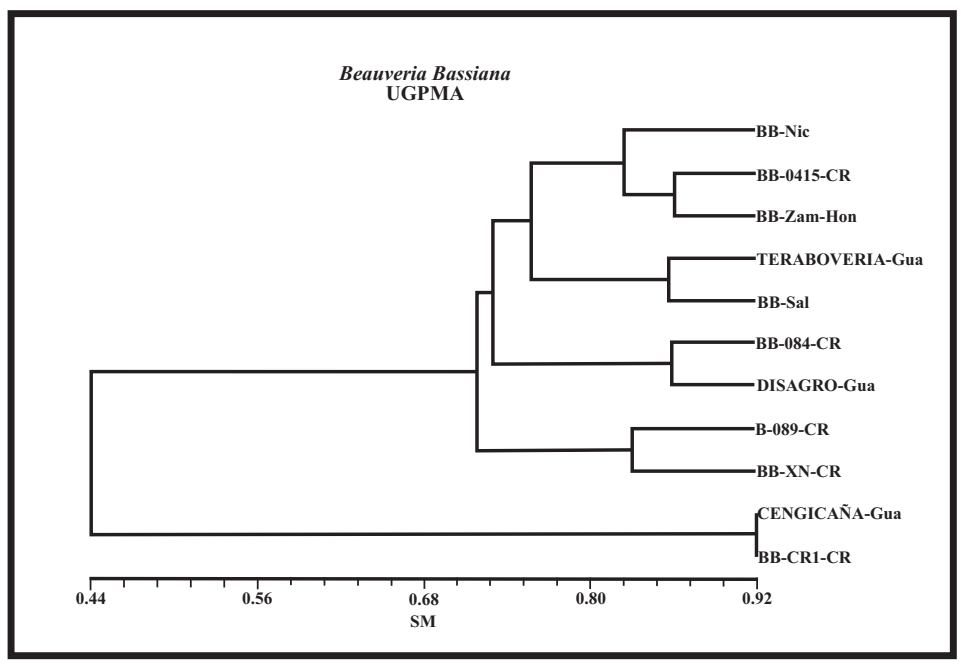

\section{Secuencia de ocho ISSR diferentes en los aislamientos de Centroamérica}

Se estudiaron ocho secuencias de ISSR en los once aislamientos procedentes de Centroamérica. El estudio de las secuencias analizadas ha puesto de manifiesto la existencia de polimorfismos de un solo nucleótido (SNPs), inserciones y deleciones (INDELS) y microsatélites (SSRs). Con los datos de estas secuencias se han obtenido las relaciones filogenéticas existentes entre aislados. Estos resultados señalan una vez más que Cengicaña y CR1 de Costa Rica están más alejados del resto de aislados. El siguiente dendrograma (Fig.7) expresa las relaciones filogenéticas obtenidas con estas secuencias de ISSR. 
Figura 7. Relaciones filogenéticas entre aislados de $B$. bassiana utilizando secuencias de microsatélites.

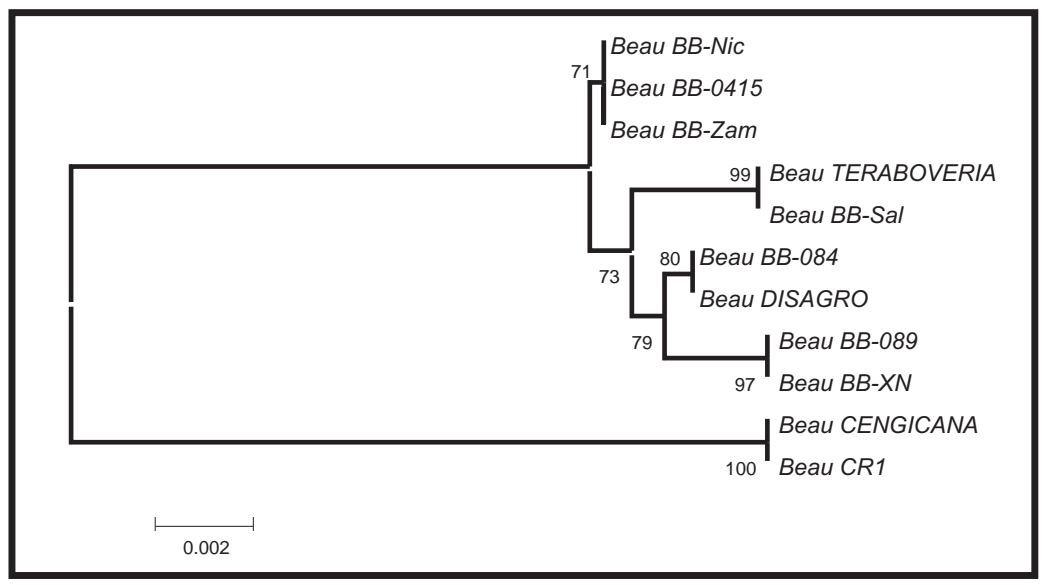

Es de notar que el dendrograma tiene la misma estructura que el obtenido con amplificaciones de ISSR, por lo que podemos concluir que estos marcadores son tan fiables como los análisis de varias secuencias a la hora de establecer relaciones filogenéticas. Además, el empleo de las secuencias de ISSR al igual que los perfiles de bandas obtenidos por amplificación de ISSR, nos han permitido identificar molecularmente todos los aislados empleados en el estudio.

Dado que BB-XN mostró un rendimiento levemente superior al resto de aislamientos bajo condiciones de campo y laboratorio; y ya que fue posible distinguirlo de los demás mediante ISSR y secuenciación de microsatélites, estos marcadores pueden ser utilizados para establecer una clara identidad del hongo con fines de explotación comercial. Es interesante que este aislado permanece como una cepa más dentro de una colección en la Universidad CATIE de Costa Rica, y parece ser más eficaz que otros aislados que están siendo comercializados en Guatemala, Honduras y el mismo Costa Rica. Por tanto, no estaría de más investigar cuál es la riqueza genética y fenotípica de los aislados locales que siguen manteniéndose almacenados como colecciones u otros que aún no han sido aislados, para luego compararlos con los productos actualmente comercializados en el Istmo.

\section{Estudios de la secuencia de SSR en los once aislados no centroamericanos}

Con los otros once aislados de procedencia extra-Centroamericana (Caribe, Estados Unidos, Europa e India) se llevó a cabo el estudio de las mismas secuencias 
de ISSR para observar su comportamiento filogenético usando los mismos marcadores. Las secuencias revelaron SNP, INDEL y microsatélites. En total se analizaron 3,586 posiciones de las que 105 fueron polimórficas y 24 solamente se observaron en un aislamiento (polimorfismos exclusivos). En el cuadro 7 se resumen los resultados obtenidos para cada una de los ocho ISSR secuenciados.

De los cinco microsatélites detectados tres fueron variables. El número de SNP por cada 100 pares de bases fue muy elevado en el ISSR Beau-1 (11,52), y en el total de las ocho secuencias fue de casi $3(2,95)$. Basándonos en la existencia de la variabilidad detectada en estas ocho secuencias hemos diseñado parejas de cebadores que permiten detectar determinados microsatélites (SSR) e inserciones/deleciones mediante PCR sin necesidad de tener que secuenciar.

Con los datos de las ocho secuencias, en estos once aislados se han obtenido las relaciones filogenéticas existentes. Los resultados arrojaron que los aislados procedentes de Cuba y Guadalupe son más cercanos entre sí, que los de India, Bulgaria y USA. Al comparar el grado de separación de los aislados centroamericanos con los de origen extra centroamericano, se sugiere la posibilidad de una estrecha relación filogenética entre los aislados de Centroamérica (Figura 7).

Estos hallazgos corroboran la idea de que a mayor distancia geográfica entre aislados, será mayor su distancia filogenética y resultará mejor la posibilidad de establecer una procedencia de los aislados.

Cuadro 7. SSR secuenciados a partir de aislados de B. bassiana no centroamericanos

\begin{tabular}{|c|c|c|c|c|c|c|c|c|c|c|c|c|}
\hline ISSR & $\begin{array}{c}\text { Tamaño } \\
(\mathbf{p b})\end{array}$ & SNP & INDEL & SSR & $\begin{array}{c}\text { SNPs/ } \\
\mathbf{1 0 0} \mathbf{p b}\end{array}$ & $\begin{array}{c}\text { INDEL/ } \\
\mathbf{1 0 0} \mathbf{p b}\end{array}$ & $\begin{array}{c}\text { SSR/ } \\
\mathbf{1 0 0} \mathbf{p b}\end{array}$ & $\begin{array}{c}\text { Exclu- } \\
\text { sivos }\end{array}$ & T\% & $\mathbf{C} \%$ & $\mathbf{A} \%$ & $\mathbf{G} \%$ \\
\hline Beau-1 & $263-266$ & 31 & 5 & 0 & 11.52 & 1.86 & 0 & 5 & 23.8 & 25.4 & 31.6 & 19.1 \\
\hline Beau-2a & $401-410$ & 20 & 9 & 0 & 4.72 & 2.12 & 0 & 2 & 22.6 & 31.7 & 19.9 & 25.8 \\
\hline Beau-2b & $472-479$ & 15 & 4 & 1 & 3.12 & 0.83 & 0.21 & 1 & 26.4 & 31.0 & 24.5 & 18.1 \\
\hline Beau-2c & $500-513$ & 19 & 9 & 0 & 3.69 & 1.75 & 0 & 8 & 24.9 & 18.3 & 25.7 & 31.1 \\
\hline Beau-3 & $563-577$ & 3 & 4 & 3 & 0.52 & 0.69 & 0.52 & 2 & 21.3 & 31.5 & 25.4 & 21.8 \\
\hline Beau-52 & $466-469-$ & 7 & 2 & 1 & 1.48 & 0.42 & 0.21 & 0 & 22.3 & 26.5 & 25.5 & 25.7 \\
\hline Beau-32 & $385-386$ & 3 & 1 & 0 & 0.78 & 0.26 & 0 & 4 & 20.5 & 24.1 & 33.6 & 21.8 \\
\hline Beau-72 & $458-463$ & 7 & 1 & 0 & 1.51 & 0.21 & 0 & 2 & 26.4 & 20.0 & 28.6 & 25.0 \\
\hline Todos & $3520-$ & 105 & 35 & $\mathbf{5}$ & $\mathbf{2 . 9 5}$ & $\mathbf{0 . 9 8}$ & $\mathbf{0 . 1 2}$ & $\mathbf{2 4}$ & $\mathbf{2 3 . 5}$ & $\mathbf{2 6 . 1}$ & $\mathbf{2 6 . 5}$ & $\mathbf{2 3 . 8}$ \\
\hline & 3557 & & & & & & & & & & & \\
\hline
\end{tabular}


Figura 7. Relaciones filogenéticas a partir de las secuencias SSR de $B$. bassiana no centroamericanos.

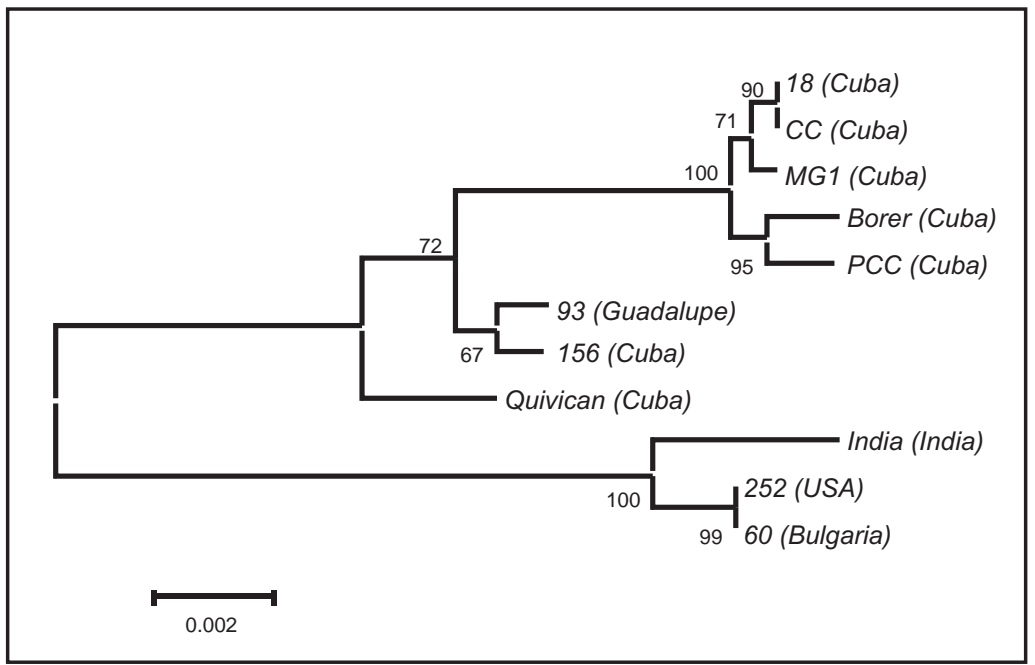

\section{CONCLUSIONES}

Diferentes marcadores moleculares han sido utilizados para detectar la variabilidad genética de Beauveria bassiana. Hasta donde sabemos, esta es la primera vez que se utilizan marcadores ISSR e ITS para el análisis filogenético de aislados de $B$. bassiana en Honduras. LOS ISSR son marcadores de fácil empleo y de alta reproducibilidad. Proveen un sistema muy eficiente como marcadores moleculares gracias a su capacidad de revelar muchas bandas informativas en una sola amplificación. Debido a esto, es una de las técnicas más utilizadas en la diferenciación de individuos de una misma especie. En este caso, quedó demostrado que los aislados obtenidos de todo el Istmo centroamericano no presentan demasiada diversidad genética y tampoco hay diferencias significativas en sus capacidades infectivas sobre la broca del café. No pudimos correlacionar de manera contundente un marcador molecular con un fenotipo superior por su patogenicidad en contra de la Broca, pero mediante SSR fue posible establecer un perfil molecular capaz de distinguir entre aislados para conferirles una identidad que pudiera ser sujeto de certificación comercial. Entre los once aislados, la BB-XN de Costa Rica parece ser la que posee un potencial más alto para su utilización como control de la broca del café en Centroamérica. Además este estudio ha permitido desarrollar marcadores moleculares relativamente fáciles de realizar y capaces de establecer la identidad genética de los aislamientos analizados. 


\section{AGRADECIMIENTOS}

Este trabajo fue financiado por las siguientes Instituciones: Dirección de Investigación Científica. DICU-UNAH; International Foundation for Science (IFS), Proyecto 3535-2; AECI - Proyecto A/016155/08. Agradecemos a todas las personas, estudiantes y autoridades universitarias que contribuyeron a la concreción de este proyecto en la Escuela Agrícola Panamericana, en el Depto. de Genética de la Universidad Complutense de Madrid y en la Escuela de Microbiología de la UNAH.

\section{REFERENCIAS}

Alves, S.B., et al., Beauveria bassiana yeast phase on agar medium and its pathogenicity against Diatraea saccharalis (Lepidoptera: Crambidae) and Tetranychus urticae (Acari: Tetranychidae). J Invertebr Pathol, 2002. 81(2): p. 70-7.

Aquino de Muro, M., et al., Molecular characterisation of Beauveria bassiana isolates obtained from overwintering sites of Sunn Pests (Eurygaster and Aelia species). Mycol Res, 2005. 109(Pt 3): p. 294-306

Aquino de Muro, M., S. Mehta, and D. Moore, The use of amplified fragment length polymorphism for molecular analysis of Beauveria bassiana isolates from Kenya and other countries, and their correlation with host and geographical origin. FEMS Microbiol Lett, 2003. 229(2): p. 249-57.

Becerra, V.M.P., C. Rojo, A. France, RAPD and ITS reveal molecular variation of Chilean populations of Beauveria bassiana. AGRICULTURA TÉCNICA (CHILE), 2007.67(2): p. 115-125.

Berretta, M.F., et al., Genotyping isolates of the entomopathogenic fungus beauveria bassiana by RAPD with fluorescent labels. J Invertebr Pathol, 1998. 71(2): p. 145-50.

Bustillo et al, Manejo integrado de la broca del café Hypothenemus hampei (Ferr.) en Colombia, in CENICAFE. 1998, CENICAFE: Chinchiná, Colombia. p. 134.

Bustillo, A.e.a., Evaluaciones de Campo con el Hongo Beauveria bassiana para el Control de la Broca del Café, Hypothenemus hampei en Colombia. 1999: Colombia.

Carvalho, A., et al., Genetic diversity and variation among botanical varieties of old Portuguese wheat cultivars revealed by ISSR assays. Biochem Genet, 2009. 47(3-4): p. 276-94.

Castrillo, L.A. and W.M. Brooks, Differentiation of beauveria bassiana isolates from the darkling beetle, alphitobius diaperinus, using isozyme and RAPD analyses. J Invertebr Pathol, 1998. 72(3): p. 190-6. 
Clark, T.B., et al., Field and laboratory studies on the pathogenicity of the fungus Beauveria bassiana to three genera of mosquitoes. J Invertebr Pathol, 1968. 11(1): p. 1-7.

Cradock, K.R. and G.R. Needham, Beauveria bassiana (Ascomycota: Hypocreales) as a management agent for free-living Amblyomma americanum (Acari: Ixodidae) in Ohio. Exp ApplAcarol, 2010.

Cruz, L.P., A.L. Gaitan, and C.E. Gongora, Exploiting the genetic diversity of Beauveria bassiana for improving the biological control of the coffee berry borer through the use of strain mixtures. Appl Microbiol Biotechnol, 2006. 71(6): p. 918-26.

Dalzoto, P.R., et al., RAPD analyses of recombination processes in the entomopathogenic fungus Beauveria bassiana. Mycol Res, 2003. 107(Pt 9): p. 1069-74.

dos Santos, H.J., Jr., et al., [Interaction of Metarhizium anisopliae (Metsch.) Sorok., Beauveria bassiana (Bals.) Vuill. and the parasitoid Oomyzus sokolowskii (Kurdjumov) (Hymenoptera: Eulophidae) with larvae of diamondback moth, Plutella xylostella (L.) (Lepidoptera: Plutellidae)]. Neotrop Entomol, 2006. 35(2): p. 241-5.

Estrada, M.E., M.V. Camacho, and C. Benito, The molecular diversity of different isolates of Beauveria bassiana (Bals.) Vuill. as assessed using intermicrosatellites (ISSRs). Cell Mol Biol Lett, 2007. 12(2): p. 240-52.

Gaitan, A.L., A. M. Valderrama, G. Saldarriaga, P. Velez, A. Bustillo, Genetic variability of Beauveria bassiana associated with Coffee Berry Borer Hypothenemus hampei and other insects. Mycol. Res, 2002. 106(11): p. 1307-1314.

Ghikas, D.V., V.N. Kouvelis, and M.A. Typas, Phylogenetic and biogeographic implications inferred by mitochondrial intergenic region analyses and ITS1-5.8SITS2 of the entomopathogenic fungi Beauveria bassiana and B. brongniartii. BMC Microbiol, 2010. 10: p. 174.

Godwin, I.D., E.A. Aitken, and L.W. Smith, Application of inter simple sequence repeat (ISSR) markers to plant genetics. Electrophoresis, 1997. 18(9): p. 15248.

Gonzales et al, Bioensayo para evaluar la patogenicidad de Beauveria bassiana (Bals.) Vuill. sobre la broca del café Hypothenemus hampei (Ferr.). Rev. Col. Entom., 1993. 19(4): p. 123-130.

Li, M., et al., [Genetic diversity of Beauveria bassiana (Bals.) Vuill. in forest ecosystem assessed by inter-simple sequence repeat (ISSR) markers]. Yi Chuan, 2006. 28(8): p. 977-83.

Liu, $\mathrm{H}$., et al., Characterization of Beauveria bassiana and Metarhizium anisopliae isolates for management of tarnished plant bug, Lygus lineolaris (Hemiptera: 
Miridae). J Invertebr Pathol, 2003. 82(3): p. 139-47.

Liu, J.N., F.R. Gui, and Z.Y. Li, Genetic diversity of the planthopper, Sogatella furcifera in the Greater Mekong Subregion detected by inter-simple sequence repeats (ISSR) markers. J Insect Sci, 2010. 10: p. 52.

Matos, M., O. Pinto-Carnide, and C. Benito, Phylogenetic relationships among Portuguese rye based on isozyme, RAPD and ISSR markers. Hereditas, 2001. 134(3): p. 229-36.

Monzon, A.J., F. Guharay, and I. Klingen, Natural occurrence of Beauveria bassiana in Hypothenemus hampei (Coleoptera: Curculionidae) populations in unsprayed coffee fields. J Invertebr Pathol, 2008. 97(2): p. 134-41.

Neelapu, N.R., et al., Molecular phylogeny of asexual entomopathogenic fungi with special reference to Beauveria bassiana and Nomuraea rileyi. Rev Iberoam Micol, 2009. 26(2): p. 129-45.

Ownley, B.H., et al., Beauveria bassiana: endophytic colonization and plant disease control. J Invertebr Pathol, 2008. 98(3): p. 267-70.

Padjama V and G Kaur, Use of the fungus Beauveria bassiana (Bals.) Vuill (Moniliales: Deuteromycetes) for controlling termites. Curr. Sci., 2001. 81(6): p. 645-647

Pashaei, S., et al., Genetic diversity in mazandaranian native cattle: a comparison with Holstein cattle, using ISSR marker. Pak J Biol Sci, 2009. 12(9): p. 717-21.

Pathan, A.A., et al., Analysis of differential gene expression in the generalist entomopathogenic fungus Beauveria bassiana (Bals.) Vuillemin grown on different insect cuticular extracts and synthetic medium through cDNA-AFLPS. Fungal Genet Biol, 2007. 44(12): p. 1231-41.

Pava-Ripoll, M., et al., Increased pathogenicity against coffee berry borer, Hypothenemus hampei (Coleoptera: Curculionidae) by Metarhizium anisopliae expressing the scorpion toxin (AalT) gene. J Invertebr Pathol, 2008. 99(2): p. 220-6.

Roddam LF, A.R., Isolation and characterization of Metarhizium anisopliae and Beauveria basssiana from subantartic macquarie island. J. Invert. Pathol, 1997. 69: p. 285-288.

Sandoval Ortiz LR, Evaluación de la eficiencia de tres productos del hongo Beauveria bassiana y Thiodan® para el control de broca del café. 1997, EscuelaAgrícola Panamericana: Tegucigalpa, Honduras. p. 43.

Smolik, M. and O. Krzysztoszek, Evaluation of genetic variability in choosen apple (Malus x domestica Borkh.) cultivars by ISSR-PCR analysis. Genetika, 2010. 46(7): p. 923-31.

Stolpovskii lu, A., et al., [The use of the ISSR-PCR method for identifying domesticated animal breeds and species, inferring their population structures, 
and assessing gene pool similarity]. Genetika, 2010. 46(6): p. 825-33.

Vaulin, O.V. and I.K. Zakharov, [Temporal dynamics and variation of multilocus ISSRPCR DNA markers in the Uman' population of Drosophila melanogasterover two decades (1984-2004)]. Genetika, 2008. 44(3): p. 359-65.

Wang, C., etal., Molecular investigation on strain genetic relatedness and population structure of Beauveria bassiana. Environ Microbiol, 2003. 5(10): p. 908-15.

Wang, S., et al., Genetic diversity and population structure among strains of the entomopathogenic fungus, Beauveria bassiana, as revealed by inter-simple sequence repeats (ISSR). Mycol Res, 2005. 109(Pt 12): p. 1364-72.

Wraight, S.P., et al., Pathogenicity of the entomopathogenic fungi paecilomyces spp. and Beauveria bassiana against the silverleaf whitefly, Bemisia argentifolii. J Invertebr Pathol, 1998. 71(3): p. 217-26. 